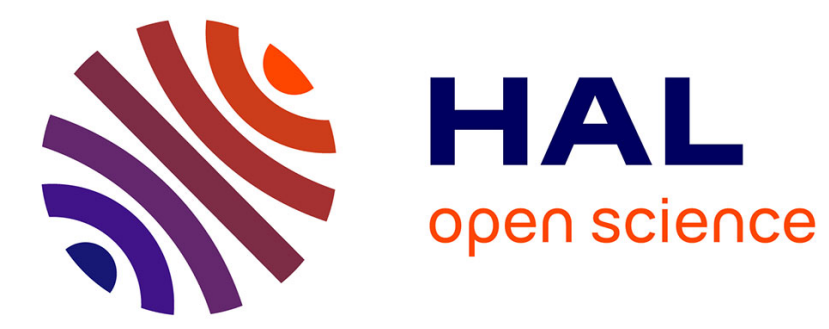

\title{
Application of a Non-Linear Damped Harmonic Analysis method to the normal modes of the Earth
}

\author{
Severine Rosat, Toshio Fukushima, Tadahiro Sato, Yoshiaki Tamura
}

\section{To cite this version:}

Severine Rosat, Toshio Fukushima, Tadahiro Sato, Yoshiaki Tamura. Application of a Non-Linear Damped Harmonic Analysis method to the normal modes of the Earth. Journal of Geodynamics, 2007, 45 (1), pp.63. 10.1016/j.jog.2007.06.001 . hal-00531877

\section{HAL Id: hal-00531877 \\ https://hal.science/hal-00531877}

Submitted on 4 Nov 2010

HAL is a multi-disciplinary open access archive for the deposit and dissemination of scientific research documents, whether they are published or not. The documents may come from teaching and research institutions in France or abroad, or from public or private research centers.
L'archive ouverte pluridisciplinaire HAL, est destinée au dépôt et à la diffusion de documents scientifiques de niveau recherche, publiés ou non, émanant des établissements d'enseignement et de recherche français ou étrangers, des laboratoires publics ou privés. 


\section{Accepted Manuscript}

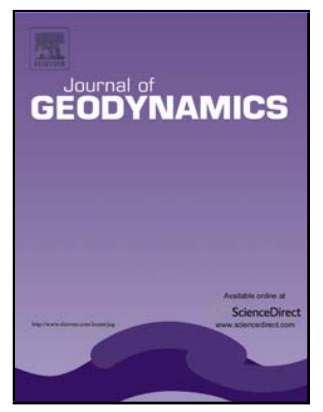

Title: Application of a Non-Linear Damped Harmonic Analysis method to the normal modes of the Earth

Authors: Severine Rosat, Toshio Fukushima, Tadahiro Sato, Yoshiaki Tamura

PII: S0264-3707(07)00049-X

DOI: doi:10.1016/j.jog.2007.06.001

Reference: GEOD 816

To appear in: Journal of Geodynamics

Received date: $\quad 11-1-2007$

Revised date: 14-5-2007

Accepted date: $\quad$ 21-6-2007

Please cite this article as: Rosat, S., Fukushima, T., Sato, T., Tamura, Y., Application of a Non-Linear Damped Harmonic Analysis method to the normal modes of the Earth, Journal of Geodynamics (2007), doi:10.1016/j.jog.2007.06.001

This is a PDF file of an unedited manuscript that has been accepted for publication. As a service to our customers we are providing this early version of the manuscript. The manuscript will undergo copyediting, typesetting, and review of the resulting proof before it is published in its final form. Please note that during the production process errors may be discovered which could affect the content, and all legal disclaimers that apply to the journal pertain. 
Table 1 Characteristics of the NLDHA and ESPRIT methods.

\begin{tabular}{|c|c|c|}
\hline & NLDHA & ESPRIT \\
\hline Computation time & Time consuming & No \\
\hline Initial estimates & Periodogram & Autoregressive model \\
\hline Model & $\begin{array}{c}\text { Polynomial trend }+ \\
\text { damped harmonic functions }\end{array}$ & Fixed a-priori \\
\hline Number of waves & Not fixed a-priori & No \\
\hline Updating of parameters at each step & Yes & Yes \\
\hline Damping estimate & Yes & No \\
\hline Initial amplitude & Yes & precise \\
\hline Starting time of excitation & No & no \\
\hline Precision of parameter estimates & most precise & yes \\
\hline Unevenly space records & &
\end{tabular}


Table 2 Estimated frequencies in $\mathrm{mHz}$ of ${ }_{0} \mathrm{~S}_{2}$ singlets from the 22-day Strasbourg SG record after the 2004 Sumatra earthquake using a Lorentzian fitting in amplitude spectrum, NLDHA and ESPRIT methods. Note that in Rosat et al. (2005) the errors attributed to the frequency estimates of ${ }_{0} \mathrm{~S}_{2}$ are based on the signal-to-noise ratio (SNR) of the signal and are not formal uncertainties.

\begin{tabular}{|c|c|c|c|c|c|}
\hline Frequency (mHz) & $\mathrm{m}=-2$ & $\mathrm{~m}=-1$ & $\mathrm{~m}=0$ & $\mathrm{~m}=1$ & $\mathrm{~m}=2$ \\
\hline Lorentzian fit (Rosat et al.2005) & 0.29997 & 0.30462 & 0.30929 & 0.31389 & 0.31845 \\
SNR errors (Rosat et al.2005) & $6.610^{-5}$ & $1.110^{-4}$ & $6.110^{-5}$ & $9.610^{-5}$ & $3.310^{-5}$ \\
Uncertainties & $1.610^{-5}$ & $1.910^{-5}$ & $1.510^{-5}$ & $2.110^{-5}$ & $1.610^{-5}$ \\
\hline NLDHA & 0.299951 & 0.304599 & 0.3092607 & 0.3138446 & 0.3184385 \\
Uncertainties & $1.510^{-6}$ & $1.610^{-6}$ & $2.510^{-7}$ & $2.610^{-7}$ & $2.810^{-7}$ \\
\hline ESPRIT & 0.29997 & 0.30460 & 0.30928 & 0.31388 & 0.3184 \\
Uncertainties & $1.710^{-5}$ & $1.210^{-5}$ & $1.810^{-5}$ & $6.210^{-5}$ & $6.410^{-4}$ \\
\hline PREM & 0.30000117 & 0.30449303 & 0.30906353 & 0.31371556 & 0.31845238 \\
\hline
\end{tabular}


Table 3 Estimated quality factors of ${ }_{0} \mathrm{~S}_{2}$ singlets from the 22-day Strasbourg SG record after the 2004

Sumatra earthquake using an amplitude decay measurement method, NLDHA and ESPRIT methods.

\begin{tabular}{|c|c|c|c|c|c|}
\hline Q & $\mathrm{m}=-2$ & $\mathrm{~m}=-1$ & $\mathrm{~m}=0$ & $\mathrm{~m}=1$ & $\mathrm{~m}=2$ \\
\hline Amplitude decay measurement & 440 & 493 & 545 & 484 & 555 \\
Uncertainties & 6 & 11 & 14 & 11 & 5 \\
\hline NLDHA & 449.3 & 481.5 & 506.7 & 457.7 & 518.7 \\
Uncertainties & 0.1 & 0.1 & 0.4 & 0.3 & 0.4 \\
\hline ESPRIT & 453.2 & 433.2 & 528.1 & 481.4 & 539.1 \\
Uncertainties & 5 & 2.7 & 4.3 & 17.8 & 12.5 \\
\hline PREM (Millot-Langet et al. 2003) & 494.6 & 501.8 & 509.3 & 517.0 & 525.0 \\
\hline
\end{tabular}


Table 4 Estimated frequencies and quality factors of ${ }_{0} S_{3}$ singlets from the 22-day Strasbourg SG record after the 2004 Sumatra earthquake using NLDHA.

\begin{tabular}{|c|c|c|c|c|c|c|c|c|}
\hline \multicolumn{2}{|r|}{${ }_{0} \mathrm{~S}_{3}$} & $m=-3$ & $m=-2$ & $m=-1$ & $\mathrm{~m}=0$ & $\mathrm{~m}=1$ & $\mathrm{~m}=2$ & $\mathrm{~m}=3$ \\
\hline \multirow{2}{*}{ NLDHA } & frequency (mHz) & $\begin{array}{l}0.4615728 \\
\pm 4.910^{-6}\end{array}$ & $\begin{array}{l}0.4642270 \\
\pm 4.910^{-6}\end{array}$ & $\begin{array}{l}0.4664168 \\
\pm 4.610^{-7}\end{array}$ & - & $\begin{array}{c}0.4707937 \\
\pm 3.810^{-6}\end{array}$ & $\begin{array}{l}0.4727150 \\
\pm 6.910^{-6}\end{array}$ & $\begin{array}{r}0.4747854 \\
\pm 1.110^{-6}\end{array}$ \\
\hline & Q & $\begin{array}{r}380.3 \\
\pm 0.04\end{array}$ & $\begin{array}{l}412.7 \\
\pm 0.1\end{array}$ & $\begin{array}{l}447.5 \\
\pm 0.5\end{array}$ & - & $\begin{array}{l}446.1 \\
\pm 0.1\end{array}$ & $\begin{array}{l}477.2 \\
\pm 0.2\end{array}$ & $\begin{array}{l}328.2 \\
\pm 0.3\end{array}$ \\
\hline $\begin{array}{l}\text { Rosat et al. } \\
\text { (2005) }\end{array}$ & frequency (mHz) & 0.46167 & 0.46424 & 0.46639 & - & 0.47084 & 0.47266 & 0.47474 \\
\hline \multirow[b]{2}{*}{ PREM } & frequency (mHz) & 0.4618 & 0.4641 & 0.4664 & 0.4686 & 0.4707 & 0.4728 & 0.4748 \\
\hline & $\begin{array}{l}\text { Q (Millot-Langet et } \\
\text { al. 2003) }\end{array}$ & 411.7 & 413.6 & 415.5 & 417.4 & 419.3 & 421.3 & 423.3 \\
\hline
\end{tabular}


Application of a Non-Linear Damped Harmonic Analysis method to the normal modes of the Earth

Severine Rosat $^{1, *}$, Toshio Fukushima ${ }^{1}$, Tadahiro Sato ${ }^{1,2}$ and Yoshiaki Tamura ${ }^{1}$

*Corresponding author

E-mail address: Severine.Rosat@oma.be

Fax: (+32) 2 374.98.22

Currently at the Royal Observatory of Belgium

Address:

Royal Observatory of Belgium

Ave. Circulaire 3

1180 BRUSSELS

BELGIUM

${ }^{1}$ National Astronomical Observatory of Japan

${ }^{2}$ Graduate School of Science, Tohoku University

\section{Abstract}

The search for weak oscillations in noisy records requires the development of analysis tools more sophisticated than the Fourier spectrum analysis. We present in this paper a new non-linear damped harmonic analysis (NLDHA) method that enables us to detect any damped harmonic signal hidden in noise and to simultaneously estimate the amplitude, frequency, phase and damping factor of the harmonic signal. We also compare this newly developed NLDHA method with a conventional autoregressive method called ESPRIT. The application of the NLDHA method to the seismic normal modes ${ }_{0} \mathrm{~S}_{2}$ and ${ }_{0} \mathrm{~S}_{3}$ excited after the 2004 Sumatra-Andaman earthquake highlights its ability to precisely recover the frequencies and quality factors of the singlets that constitute these two seismic modes. The application of the NLDHA in the sub-seismic frequency band reveals the presence of some unmodelled waves but no detection of the translational oscillation of the inner core has been claimed.

Keywords: non-linear harmonic analysis, normal modes, superconducting gravimeters, Slichter triplet

\section{Introduction}


The density structure in the deep Earth is poorly constrained, especially inside the core. Knowledge of the density structure inside the core can be achieved by the observation of the surface gravity effect of the core modes and of the translation of the solid inner core in the fluid outer core, the so-called Slichter triplet (Slichter, 1961). The period of the Slichter triplet is directly dependent on the density jump at the inner core boundary (ICB) as the main restoring force is the Archimedean force. The PREM (Dziewonski and Anderson, 1981) reference model gives a density jump at the ICB of $600 \mathrm{~kg} / \mathrm{m}^{3}$ while recent observations by Masters and Gubbins (2003), and Koper and Pyle (2004), reached diverging conclusions, respectively that the density jump at ICB should be greater or smaller than the PREM predicted value. Moreover a convincing detection of this harmonic degree one normal mode could also constrain the viscosity at the ICB (e.g. Smylie and McMillan, 1998 and 2000; Rieutord, 2002). However, many attempts using spectral methods and stacking processes have not yet led to the reliable detection of the surface gravity signature of the free oscillation of the inner core (Jensen et al., 1995; Hinderer et al., 1995; Rosat et al., 2003 and 2006; Guo et al. 2006a and 2006b) while the unique observation by Smylie (1992) and Smylie et al. (1993) has been confirmed by Courtier et al. (2000). However, Rieutord (2002) and Rogister (2003) have demonstrated that this unique claim of detection is irrelevant to the theoretical predictions.

As the problem is to detect weak signals buried in larger amplitude geophysical phenomena and with unknown parameters (frequency, damping factor, and excitation source), we have to use more sophisticated tools than the usual spectral methods. As the period of the Slichter triplet is poorly constrained, the frequency must not be fixed a-priori. The process of excitation of the free oscillation of the inner core is also poorly known. Therefore the damping factor must also be an estimated parameter and not fixed a-priori. That highlights our motivation to develop a non-linear damped harmonic analysis (NLDHA), which is based on the non-linear harmonic analysis (NLHA) developed by Harada (2003). The NLHA method was successfully applied to the estimation of the time ephemeris of the Earth (Harada and Fukushima, 2003) as well as to the determination of planetary precession (Harada and Fukushima, 2004). 
Here we propose a generalization of the method to damping oscillations and a first application to the seismic normal modes of the Earth that induced time-varying gravity signals recorded on the Earth's surface by Superconducting Gravimeters (SGs) of the Global Geodynamics Project (GGP; Crossley et al., 1999) after the 2004 Sumatra-Andaman earthquake. SGs have proved to be well-suited for the study of the long-period seismic and sub-seismic modes (e.g. Freybourger et al., 1997; Van Camp, 1999; Widmer-Schnidrig, 2003; Rosat et al., 2004). The precise estimation of the frequencies and damping of the seismic modes of the Earth below $1 \mathrm{mHz}$, is fundamental as they are very sensitive to the density structure inside the Earth. In particular, their frequency splitting is directly linked to the 1D-density structure (Widmer-Schnidrig, 2003). Moreover their splitting possesses high sensitivity to the 3D-density structure in the Earth's mantle and core. Therefore these modes are a mean to improve the density profile inside the Earth without any tradeoff with elastic parameters. Finally, an application of the NLDHA to the SG gravity residuals at Strasbourg (France) and Canberra (Australia) in the sub-seismic frequency range is performed in the search for the surface gravity effect of the Slichter modes.

\section{Description of NLDHA method}

We extended Harada's NLHA (Harada, 2003; Harada and Fukushima, 2003 and 2004) so as to be applicable to time series containing unknown numbers of damping oscillations with unknown frequencies and damping factors. The extended method tries to minimize $\Phi$, the weighted sum of the square of residuals of the given data after subtracting a polynomial trend and multiple damping oscillations, defined by:

$$
\Phi(p, s, c, \omega, \lambda) \equiv \frac{1}{2} \sum_{i=1}^{N} w_{i}\left[\sum_{j=0}^{L} p_{j} t_{i}^{j}+\sum_{k=1}^{M}\left\{\exp \left(-\lambda_{k} t_{i}\right)\left(s_{k} \sin \omega_{k} t_{i}+c_{k} \cos \omega_{k} t_{i}\right)\right\}-f_{i}\right]^{2},
$$

where $N$ is the number of data, $f_{i}$ is the $i$-th datum obtained at the time $t_{i}$, and $w_{i}$ is its weight; $L$ is the order of the trend polynomial, $p_{j}$ is its $j$-th coefficient; $M$ is the number of damping oscillations, 
$s_{k}$ and $c_{k}$ are the coefficients of the sine and cosine parts of the $k$-th oscillation component, the angular frequency and the damping factor of which are $\omega_{k}$ and $\lambda_{k}$, respectively. Note that the introduction of the data weights enables NLDHA to deal with records containing some gaps by attributing a weight of zero to the missing samples.

The outline of the method is roughly the same as that of Harada's method (Harada, 2003). Once the non-linear parameters, $\omega_{k}$ and $\lambda_{k}$, are all fixed, the other linear parameters, $p_{j}, s_{k}$, and $c_{k}$, are optimally determined by the method of weighted linear least squares. In this sense, the optimal linear parameters, $\underline{p}_{j}, \underline{s}_{k}$, and $\underline{c}_{k}$, can be regarded as functions of the non-linear parameters. Then we regard the objective function $\Phi$ as a function of the non-linear parameters only:

$$
\underline{\Phi}(\omega, \lambda) \equiv \Phi(\underline{p}(\omega, \lambda), \underline{s}(\omega, \lambda), \underline{c}(\omega, \lambda), \omega, \lambda) .
$$

Thus our problem reduces to a non-linear least squares problem, which is effectively solved by a quasi-Newton method such as the well-known BFGS (Broyden-Fletcher-Goldfarb-Shannon) method (Press et al., 1992). Therefore the issue is reduced to the questions of how to determine the optimal number of damping oscillations, $M$, and how to find suitable initial guess of the non-linear parameters.

We solve the first issue by the step-by-step approach, namely increasing $M$ one by one starting from 0 as was done in Harada's NLHA (Harada, 2003). This iterative approach is also similar to the iterative Fourier transform method used by Guo et al. (2005) in the search for the inner core wobble in Earth's polar motion data. As for the second issue, we pick up a candidate pair of frequency and damping factor which maximizes the decrement of the objective function in the two-dimensional search of parameters in the range pre-assigned. Note that we simplified the procedure of the search by conducting not the full two-dimensional but the alternative search in the sense of frequency-first. The initial frequency is obtained from the power spectrum of the signal for the zero-damping factor. When the frequency has been finely estimated, the damping factor is then searched. The initial damping value is obtained from the maximum of the damping power spectrum estimated at the frequency determined in the previous fine search of frequency. The power 
spectrum in the damping factor domain is uni-modal, namely containing only one maximum (cf. Fig. 1). For each direction, in the frequency or in the damping factor, we followed a three-stage approach (1) a coarse but comprehensive search, (2) a refined but still comprehensive search, both based on the quasi-Newton method implemented with the BFGS algorithm, and (3) a parabola fitting near the optimum. The final stage to find the global minimum of $\Phi$ is conducted by the Brent's method using the golden rule section (Brent, 1973). The full algorithm is sketched in Fig. 2. For the optimization subroutines used in NLDHA, the reader may refer to the Numerical Recipes (Press et al., 1992) Chapters 10.1 and 10.7, respectively for the golden section search of a local minimum and for the BFGS optimization of the quasi-Newton method.

The NLDHA method is different from the well-known auto-regressive Prony's method, discovered by Prony in 1795 (Prony, 1795) during the long-time work to compute numerical tables of sine and cosine, and its modifications (Osborne 1975, Smyth 1985, Osborne and Smyth 1995) in the sense that (1) the time of sampling can be arbitrary, (2) the secular trend can be determined simultaneously, (3) the effect of data weight is considered, and (4) the optimal number of damping oscillations is also estimated. Note that Prony's method is applicable only to evenly-sampled data. Prony's method and its modern variation were re-discovered independently many times, for example, the "Sompi" method (Hori et al. 1989, Kumazawa et al. 1990, Imanishi et al. 1992), which is now popular in geophysics. Recently the ESPRIT method (Roy 1987, Roy and Kailath 1989), which is a variation of Prony's method and originally established in the field of acoustic signal processing, seems to be better than the modified Prony's method in analyzing transient phenomena in electric power systems (Dafis et al. 2002). The ESPRIT method requires fixing apriori the model order, i.e. the number of harmonic signals to be analyzed. However, based on the estimation error (Badeau et al. 2004) for instance, it is possible to evaluate a-posteriori the optimal model order. The characteristics of the NLDHA and the auto-regressive ESPRIT methods are compared in Table 1. 
A first application of the NLDHA method to the seismic normal modes excited after the 2004 Sumatra-Andaman earthquake is proposed in the next section. The seismic normal modes are well-known decaying waves; therefore they are useful to test our NLDHA method and to compare it with more conventional analyses.

\section{Application to the seismic modes ${ }_{0} S_{2}$ and ${ }_{0} S_{3}$ after the 2004 Sumatra-Andaman earthquake}

SGs have proved to be well suited for the study of the seismic modes below $1 \mathrm{mHz}$ and the seismic mode analysis after the $\mathrm{M}_{\mathrm{w}}=9.3$ Sumatra-Andaman earthquake on 2004 December 26 has confirmed their high quality (Rosat et al., 2005). Contrary to the Slichter triplet, the frequencies and Q-values of the seismic modes are well-known from many previous studies based on seismometer data (e.g. He and Tromp, 1996) or SG data (e.g. Roult et al., 2006). Therefore the analysis of the seismic modes presents a great opportunity to test the NLDHA method.

In the following, we use 22-day SG data recorded at the Strasbourg (ST, France) station after the 2004 Sumatra event. The gravity records have been corrected for the local tides and atmospheric pressure effect through a nominal barometric admittance of $-3 \mathrm{~nm} / \mathrm{s}^{2} / \mathrm{hPa}$. The atmospheric pressure reduction is necessary to reduce the noise level at these frequencies (e.g. Zürn and Widmer, 1995).

In order to reduce the computation time of NLDHA, we limit the analysis to the frequency band $0.28-0.34 \mathrm{mHz}$ by pre-processing the data with a band-pass filter. This frequency range contains the spheroidal seismic mode ${ }_{0} \mathrm{~S}_{2}$ only. The second subscript corresponds to the spherical harmonic degree $l=2$ and the first figure is the radial number $n=0$ in the notation ${ }_{n} S_{l}$ for the spheroidal modes. Because of the Earth's rotation and ellipticity, each mode is split into $2 l+1$ singlets having distinct frequencies. Thus ${ }_{0} \mathrm{~S}_{2}$ is split into 5 frequencies. The amplitude spectrum of ${ }_{0} \mathrm{~S}_{2}$ is represented in the upper plot of Fig. 3 (a). The reconstructed signals and the residuals obtained with NLDHA and with ESPRIT are plotted in Fig. 3 (a). We have also plotted the RMS residuals after each step of the NLDHA in Fig. 3 (b). The sudden and clear drop after the $5^{\text {th }}$ step, 
i.e. after the analysis of five decaying waves, indicates that all the meaningful harmonic signals have been detected and that only noise is remaining.

A comparison of the frequency estimates after the 2004 December 26th Andaman-Sumatra earthquake using a standard Lorentzian fitting of an amplitude spectrum (Rosat et al., 2005) with the results of the NLDHA and ESPRIT methods shows a perfect similitude within the error bars (cf. Table 2). The errors computed by Rosat et al. (2005) are based on the signal to noise ratio of each singlet as proposed by Dahlen (1982), and thus do not reflect the method uncertainty. So we have also estimated the uncertainties of the Lorentzian fitting. The estimated quality factors (Table 3) are also in good agreement between the amplitude decay measurement and NLDHA methods. It is worth to note that the NLDHA method gives lower uncertainties on the estimated parameters of ${ }_{0} \mathrm{~S}_{2}$ seismic mode. The predicted frequencies and Q values of ${ }_{0} \mathrm{~S}_{2}$ for a PREM model are also given in Table 3.

We have also applied the NLDHA method to the same SG data but band-pass filtered between the corner frequencies 0.2 and $0.5 \mathrm{mHz}$. The wider band enables to estimate precisely the 5 singlets of ${ }_{0} \mathrm{~S}_{2}$ and also to recover the 6 singlets of ${ }_{0} \mathrm{~S}_{3}$ (Fig. 4 (a)). Indeed there should be 7 singlets for this harmonic degree 3 seismic mode, but because of the geographical position of ST site with respect to the earthquake source, the central singlet corresponding to $\mathrm{m}=0$ at the PREM frequency $0.4686 \mathrm{mHz}$, cannot be observed at ST. In that case, the RMS residuals do not indicate any drop up to the final step (Fig. 4 (b)). The process stops at the step 17 by itself as it cannot find any more harmonic signal to analyze. Although the NLDHA succeeds to find the harmonic signal corresponding to $\mathrm{m}=2$ and to estimate the frequency and damping factor, it fails computing the uncertainties of these non-linear parameters. The reason is that the frequency resolution is too poor to distinguish the singlet $\mathrm{m}=2$ from the singlet $\mathrm{m}=1$, so the Cholesky decomposition used to compute the inverse matrix of the uncertainties fails (the matrix becomes singular). We have resolved that problem by using a Singular Value Decomposition instead of the Cholesky decomposition when the latter fails. The results of NLDHA obtained for ${ }_{0} \mathrm{~S}_{3}$ are compared with the 
PREM predictions by Millot-Langet et al. (2003) in Table 2 for the frequencies and in Table 3 for the quality factors.

We have demonstrated with this example that NLDHA can analyze a very small oscillation hardly distinguishable from a larger harmonic signal and give a good estimate of its parameters. Of course to increase the quality of the parameter estimates we could improve the frequency resolution of the ${ }_{0} \mathrm{~S}_{3}$ seismic mode by taking longer data series, when they are available, in a similar way than when doing a spectral analysis.

To conclude this part, the NLDHA method performs well in the analysis of the seismic normal modes of the Earth. In the following section, we will show its application to the search for the Slichter triplet in the sub-seismic frequency band.

\section{Application to the search for the Slichter modes in the sub-seismic frequency band}

The mechanism of excitation of the Slichter modes is not well known. It could be excited by a strong earthquake (e.g. Rosat, 2006), randomly excited by some turbulent flow in the fluid outer core and/or by the variable motions of the atmosphere and oceans. We suppose that the Slichter modes are continuously excited or are slowly damping, so that they are statistically well represented in a year of SG gravity records, in order to be detectable by the NLDHA. We consider the SG gravity records at the Canberra (Australia) and Strasbourg (France) SG sites. These SG sites have been chosen because of their low noise level in the frequency range considered (Rosat et al. 2004) and because of their nearly opposite geographical location. Before applying the NLDHA method, we have corrected the gravity data for the local tides and the atmospheric pressure effect. The obtained residuals have then been band-pass filtered between the corner frequencies $0.02 \mathrm{mHz}$ and $0.08 \mathrm{mHz}$. That frequency range covers all the possible periods for the Slichter modes predicted or observed by the previous works (e.g. Smylie, 1992, Courtier et al. 2000, Rieutord, 2002, Rogister, 2003). 
The NLDHA analysis detects the tidal waves as well as the diurnal harmonics of the atmospheric mode $\mathrm{S} 1$ remaining in the SG data. The reconstructed signal and the final residuals are plotted in Fig. 5 (a). The diurnal harmonics of S1 and its overtones (from S2 to S7) are clearly observed. Moreover, the main frequencies of the non-linear tides (MN4, M4, SN4, MS4 and MK4 for the quart diurnal band; 2MN6, M6, MSN6, 2MS6, 2MK6, 2SM6 and MSK6 for the tidal band around 6h) are indicated. The RMS amplitudes of the residuals at each step are plotted in Fig. 5 (b). The amplitude spectra of the residuals at every five steps from 1 to 50 are also plotted in Fig. 5 (c). Notice a spectral peak in the Canberra data found at the frequency $0.0736 \mathrm{mHz}$ with a negative Qfactor equal to -37051 and an amplitude of $0.008 \mathrm{~nm} / \mathrm{s}^{2}$, and a spectral peak in the Strasbourg SG data found at the frequency $0.0774 \mathrm{mHz}$ with $\mathrm{Q}=20810$ and an amplitude of $0.003 \mathrm{~nm} / \mathrm{s}^{2}$ with a damping-rate of about 9 years. Note that, as discussed by Zürn and Rydelek (1991), it is possible to obtain a negative Q-value when the system has a very large Q and the signal amplitude is small. Another spectral peak with amplitude of $0.01 \mathrm{~nm} / \mathrm{s}^{2}$ emerges clearly above the noise level in the Canberra residuals at the frequency $0.0655 \mathrm{mHz}$ with an estimated Q-factor of 119860 giving a damping rate of about 58 years. This could be also an actual geophysical signal. However we have not enough evidence for the moment to conclude.

The frequencies detected by Courtier et al. (2000) and attributed to the Slichter triplet were 0.069186, 0.073767 and $0.077544 \mathrm{mHz}$, with quality factors between 100 and 400 (Smylie, 1992). Crossley et al. (1991) have shown that the damping due to the seismic anelasticity of the inner core and mantle has a Q-value of the order of 5000 with a corresponding damping time of 400 days. The damping due to the outer core viscosity has been formulated by Smylie and McMillan (1998) and also by Rieutord (2002). The estimates of the dynamic viscosity range from $1.610^{-2}$ Pa.s using laboratory experiments (Rutter et al. 2002), giving a Q-value of the order of $10^{7}$, to $1.210^{11}$ Pa.s (Smylie and McMillan, 2000) using the claimed Slichter modes of Courtier et al. (2000) giving a Qvalue less than 10. Mathews and Guo (2005) have proposed an upper limit of $1.710^{5} \mathrm{~Pa} . \mathrm{s}$ using nutation data corresponding to a Q-value of 5000. The magnetic damping of the inner core has been 
studied by Buffet and Goertz (1995) who have shown that the Q-value should be between $5.810^{5}$ and 2200 for a magnetic field ranging from 0.0005 to $0.001 \mathrm{~T}$.

Since the expected triplet for the Slichter modes has not been commonly detected at the two SG sites Strasbourg and Canberra, we cannot say that the Slichter triplet has been clearly detected from the present analysis. However, the obtained signals close to the proposed frequencies of the Slichter triplet show a very large Q-value (between 20000 and 120000) suggesting a very low viscosity of the fluid core - if these signals can be attributed to the Slichter modes and if we consider that the main damping is due to the viscosity of the fluid outer core - and a very small amplitude of these waves as suggested from the theoretical studies.

Besides the S1 wave and its over-tones observed in the residual time series data indicate a necessity to improve the accuracy of the atmospheric pressure correction. To strengthen the reliability of the detection of the core modes and the Slichter modes, further study is needed; particularly using an extension the NLDHA to combine several datasets should be considered (e.g. expanding the present NLDHA algorithm so that it can treat many SG data sets simultaneously).

\section{Conclusion}

The advantages of the NLDHA method reside in the complete analysis of the signal without fixing a-priori the number of waves, a highly precise estimation of the parameters values and the application to unevenly spaced datasets.

We have demonstrated that the NLDHA is well suited to the precise analysis of the Earth's normal modes. The NLDHA will be also very useful to detect and analyze the time-varying gravity record in the search for the weak oscillations of the core and inner core. In particular, a multistation extension of the NLDHA would be required to stack several SG records and search for the global signature of the translational motion of the inner core. This research will be the aim of a future work.

\section{Acknowledgments}


S. Rosat was supported by the Japan Society for the Promotion of Science during this study. This research is partly funded by the Japan Society for the Promotion of Science (JSPS) Grants-in-Aid for scientific research (B) 16340134.

\section{Bibliography}

Badeau, R., David, B and Richard, G., 2004. Selecting the modeling order for the ESPRIT high resolution method: an alternative approach, in Proc. of ICASSP'04, Montreal, Canada, May 1721, vol. II, 1025-1028.

Brent, R.P., 1973. Algorithms for Minimization without Derivatives, (Englewood Cliffs, NJ: Prentice-Hall), Chapter 5.

Buffett, B.A. \& Goertz, D.E., 1995. Magnetic damping of the translational oscillations of the inner core, Geophys. J. Int., 120, 103-110.

Courtier, N., Ducarme, B., Goodkind, J., Hinderer, J., Imanishi, Y., Seama, N., Sun, H., Merriam, J., Bengert, B. and Smylie, D.E., 2000. Global superconducting gravimeter observations and the search for the translational modes of the inner core, Phys. Earth Planet. Int., 117, 3-20.

Crossley, D., Hinderer, J. \& Legros, H., 1991. On the excitation, detection and damping of core modes, Phys. Earth Planet. Int., 68, 97-116.

Crossley, D., Hinderer, J., Casula, G., Francis, O., Hsu, H.T., Imanishi, Y., Jentzsch, G., Kääriäinen, J., Merriam, J., Meurers, B., Neumeyer, J., Richter, B., Shibuya, K., Sato, T., Van Dam, T., 1999. Network of superconducting gravimeters benefits a number of disciplines, EOS, $80,11,121 / 125-126$.

Dafis, C.J., Nwankpa, C.O., and Petropulu, A. 2002, Proceedings of the $14^{\text {th }}$ PSCC held at Sevilla on 24-28 $8^{\text {th, }}$ June, 2002, Session 28, Paper 2

Dahlen, F.A., 1982. The effect of data windows on the estimation of free oscillations parameters, Geophys. J. R. Astron. Soc., 69: 537-549.

Dziewonski A.M. and D.L. Anderson, 1981. Preliminary Reference Earth Model, Phys. Earth 
Planet. Int., 25, 297-356.

Freybourger, M., Hinderer, J. and Trampert, J., 1997. Comparative study of superconducting gravimeters and broadband seismometers STS-1/Z in subseismic frequency bands, Phys. Earth Planet. Int., 101, 203-217.

Fukao, Y. and Suda, N., 1989. Core modes of the Earth's free oscillations and structure of the inner core, Geophys. Res. Lett., 16 (5), 401-404.

Guo, J.Y., Greiner-Mai, H. and Ballani, L., 2005. A spectral search for the inner core wobble in Earth's polar motion, J. Geophys. Res., 110, B10402, doi:10.1029/2004JB003377.

Guo, J.Y., Dierks, O., Neumeyer, J., and Shum, C.K., 2006a. Weighting algorithms to stack superconducting gravimeters data for the potential detection of the Slichter modes, J. of Geodyn., 41, 326-333.

Guo, J.Y., Dierks, O., Neumeyer, J., and Shum, C.K., 2006b. A search for the Slichter modes in superconducting gravimeter records using a new method, J. of Geodyn., under press.

Harada, W., 2003. A method of non-linear harmonic analysis and its application to dynamical astronomy, Master's thesis, Univ. Tokyo.

Harada, W. and Fukushima, T., 2003. Harmonic decomposition of time ephemeris TE405, Astron. J., 126, 2557-2561.

Harada, W. and Fukushima, T., 2004. A new determination of planetary precession, Astron. J., 127, 531-538.

He, X., and Tromp, J., 1996, Normal-mode constraints on the structure of the mantle and core, J. Geophys. Res., 101, 20,053--20,082.

Hinderer J., D. Crossley and O. Jensen, 1995. A search for the Slichter triplet in superconducting gravimeter data, Phys. Earth and Planet. Int., 90, 183-195.

Hori, S., Fukao, Y., Kumazawa, M., Furumoto, M. and Yamamoto, A., 1989. A new method of spectral analysis and its application to the Earth's free oscillations: the "Sompi" method, J. Geophys. Res., 94, 7535-7553. 
Imanishi, Y., Sato, T., Kumazawa, M., Ooe, M. and Tamura, Y., 1992. Observation of seismic core modes from a superconducting gravimeter record, Phys. Earth and Planet. Int., 72, 249-263.

Jensen O., J. Hinderer and D. Crossley, 1995. Noise limitations in the core-mode band of superconducting gravimeter data, Phys. Earth and Planet. Int., 90, 169-181.

Koper, K.D. and M.L. Pyle, 2004. Observations of PkiKP/PcP amplitude ratios and implications for Earth structure at the boundaries of the liquid core, J. Geophys. Res., 109, B03301, doi :10.1029/2003JB002750.

Kumazawa, M., Imanishi, Y., Fukao, Y., Furumoto, M. and Yamamoto, A., 1990. A theory of spectral analysis based on the characteristic property of a linear dynamic system, Geophys. J. Int., 101, 613-630.

Masters, G. and D. Gubbins, 2003. On the resolution of the density within the Earth, Phys. Eart Planet. Int., 140, 159-167.

Mathews, P.M. and Guo, J.Y., 2005. Visco-electromagnetic coupling in precession-nutation theory, J. Geophys. Res., 110 (B2), B02402.doi: 10.1029/2003JB002915.

Millot-Langet, R., Clevede, E., Lognonne, P., 2003. Normal modes and long period seismograms in a 3D anelastic elliptical rotating Earth. Geophys. Res. Lett. 30, 1202-1206.

Osborne, M.R. 1975, SIAM J. Numer. Analys., 12, 571.

Osborne, M. R., and Smyth, G. K., 1995. A modified Prony algorithm for exponential function fitting, SIAM J. Sci. Statist. Comput. 16, 119-138.

Press, W.H., Teukolsky, S.A., Vetterling, W.T., Flannery B.P., 1992, Numerical Recipes in FORTRAN: the Art of Scientific Computing, 2nd Ed, Cambridge: Cambridge Univ. Press

Prony (de), Baron Gaspard Riche, 1795. Essai experimental et analytique: sur les lois de la dilatabilité de fluides élastique et sur celles de la force expansive de la vapeur de l'alkool, à différentes températures. Journal de l'École Polytechnique, volume 1, cahier 22, 24-76.

Rieutord, M., 2002. Slichter modes of the Earth revisited, Phys. Earth Planet. Int., 131, 269-278. 
Rogister Y., 2003. Splitting of seismic free oscillations and of the Slichter triplet using the normal mode theory of a rotating, ellipsoidal Earth, Phys. Earth Planet. Int., 140, 169-182.

Rosat, S., 2006. Optimal Seismic Source Mechanisms to Excite the Slichter Mode, J. of Geodesy, under press.

Rosat S., J. Hinderer, D. Crossley and L. Rivera, 2003. The search for the Slichter mode: comparison of noise levels of superconducting gravimeters and investigation of a stacking method, Phys. Earth Planet. Int., 140, 183-202.

Rosat, S., J. Hinderer, D. Crossley and J.P. Boy, 2004. Performance of superconducting gravimeters from tides to long-period seismology, J. of Geodyn, 38, 3-5, 461-476.

Rosat, S., Sato, T., Imanishi, Y., Hinderer, J., Tamura, Y., McQueen, H., Ohashi, M., 2005. High resolution analysis of the gravest seismic normal modes after the $2004 \mathrm{M}_{\mathrm{w}}=9$ Sumatra earthquake using superconducting gravimeter data, Geophys. Res. Lett., 32, L13304, doi:10.1029/2005GL023128.

Rosat, S., Rogister, Y., Crossley, D. and Hinderer, J., 2006. A Search for the Slichter Triplet with Superconducting Gravimeters: Impact of the Density Jump at the Inner Core Boundary, J. of Geodyn., 41, 296-306.

Roult, G., Rosat, S., Millot-Langet, R., Clévédé, E. and Hinderer, J., 2006. New determinations of Q quality factors and eigenfrequencies for the whole set of singlets of the Earth's normal modes 0S0,0S2,0S3 and 2S1 using SG data from the GGP network, J. of Geodyn., 41, 345-357.

Roy, R. 1987, "ESPRIT-Estimation of signal parameters via rotational imvariamce techniques”, Ph.D Thesis, Stanford Univ.

Roy, R. and Kailath, T., 1989. “ESPRIT-Estimation of Signal Parameters via Rotational Invariance Techniques”, IEEE Trans. Acous., Speech, Signal Processing, 37 (7).

Rutter, M.D., Secco, R.A., Uchida, T., Liu, H., Wang, Y., Rivers, M.L. \& Sutton, S.R., 2002. Towards evaluating the viscosity of the Earth's outer core: An experimental high pressure study of liquid Fe-S (8.5 wt.\% S), Geophys. Res. Lett., 29 (8), 1217. doi: 10.1029/2001GL014392. 
Slichter, L.B., 1961. The fundamental free mode of the Earth's inner core, Proc. Nat. Acad. Sci., 47(2), 186-190.

Smylie, D. E., 1992. The Inner Core Translational Triplet and the Density Near Earth's Center, Science, 255, 1678-1682.

Smylie, D. E., Hinderer, J., Richter, B. and Ducarme, B., 1993. The product spectra of gravity and barometric pressure in Europe, Phys. Earth Planet. Int., 80, 135-157.

Smylie, D. E. and McMillan D. G., 1998: Viscous and rotational splitting of the translational oscillations of Earth's solid inner core, Phys. Earth planet. Int., 106, 1-18.

Smylie D. E. and McMillan D. G., 2000: The inner core as a dynamic viscometer, Phys. Earth Planet. Int., 117, 71-79.

Smyth, G. K., 1985. Coupled and Separable Iterations in Nonlinear Estimation, $\mathrm{PhD}$ thesis, Canberra, Australian National University.

Van Camp, M., 1999. Measuring seismic normal modes with the GWR C021 superconducting gravimeter, Phys. Earth Planet. Int., 116, 81-92.

Widmer-Schnidrig, R., 2003. What can Superconducting Gravimeters contribute to normal mode seismology? Bull. Seism. Soc. Am., 93 (3), 1370-1380.

Zürn, W. and P.A. Rydelek, 1991. Investigation of the "Nearly diurnal free wobble" - resonance in individual tidal records, in: Kakkuri, J. (Ed.), Proceedings of the 11 International Symposium on Earth Tides, Helsinki, 521-530.

Zürn, W., and R. Widmer, 1995. On noise reduction in vertical seismic records below $2 \mathrm{mHz}$ using local barometric pressure, Geophys. Res. Lett., 22, 3537-3540.

\section{Figure caption}

Fig. 1 Example of a damping power spectrum.

Fig. 2 NLDHA algorithm. 
Fig. 3 NLDHA and ESPRIT method applied to the time-varying gravity record of the SG at Strasbourg after the Sumatra event. (a) From top to bottom, amplitude spectrum of the SG data, amplitude spectrum of the reconstructed signal and amplitude spectrum of the residuals (original signal minus the reconstructed damped oscillations) obtained from the NLDHA (solid line) and ESPRIT (dotted line) analysis results. The data have been band-pass filtered between the corner frequencies 0.28 and $0.34 \mathrm{mHz}$ before applying the NLDHA and ESPRIT. (b) Residual amplitude (RMS) after each step of the NLDHA. Note that the step number corresponds to the number of selected waves.

Fig.4 Results of the NLDHA method applied to the time-varying gravity record of the SG at Strasbourg after the Sumatra event. (a) From top to bottom, the amplitude spectra of the SG data, of the reconstructed signal and of the residuals (original signal minus the reconstructed damped oscillations) obtained from the NLDHA analysis results are plotted. The data have been band-pass filtered between the corner frequencies 0.2 and $0.5 \mathrm{mHz}$ before applying the NLDHA. (b) Residual amplitude (RMS) after each step of the NLDHA. Note that the step number corresponds to the number of selected waves.

Fig. 5 Results of the NLDHA method applied to the one-year time-varying gravity record of the Canberra (Australia) and Strasbourg (France) SG sites, respectively on the left-hand and right-hand sides. (a) From top to bottom, the amplitude spectra of the SG signal, of the reconstructed signal and of the residuals (original signal minus the reconstructed damped oscillations) obtained from the NLDHA analysis results are plotted. (b) Residual amplitude (RMS) after each step of the NLDHA. (c) Amplitude spectrum of the residuals after every five steps from 1 to 50. The diurnal harmonics of the thermal atmospheric wave S1 from S2 to S7 are indicated in vertical dashed and dotted lines and the non-linear tides around 4 and $6 \mathrm{~h}$ are indicated in vertical dotted lines. 


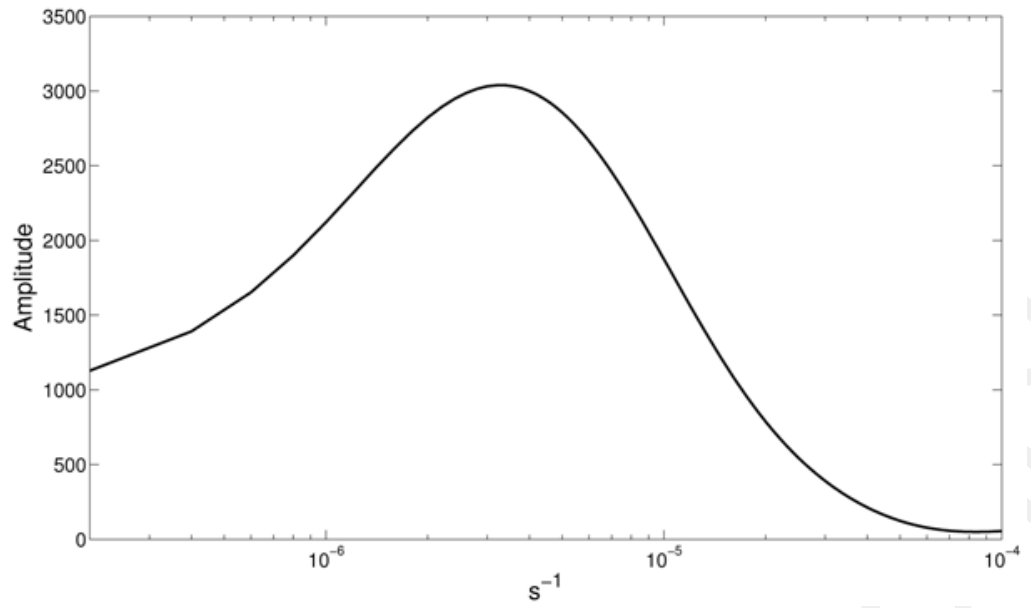

Fig.1 


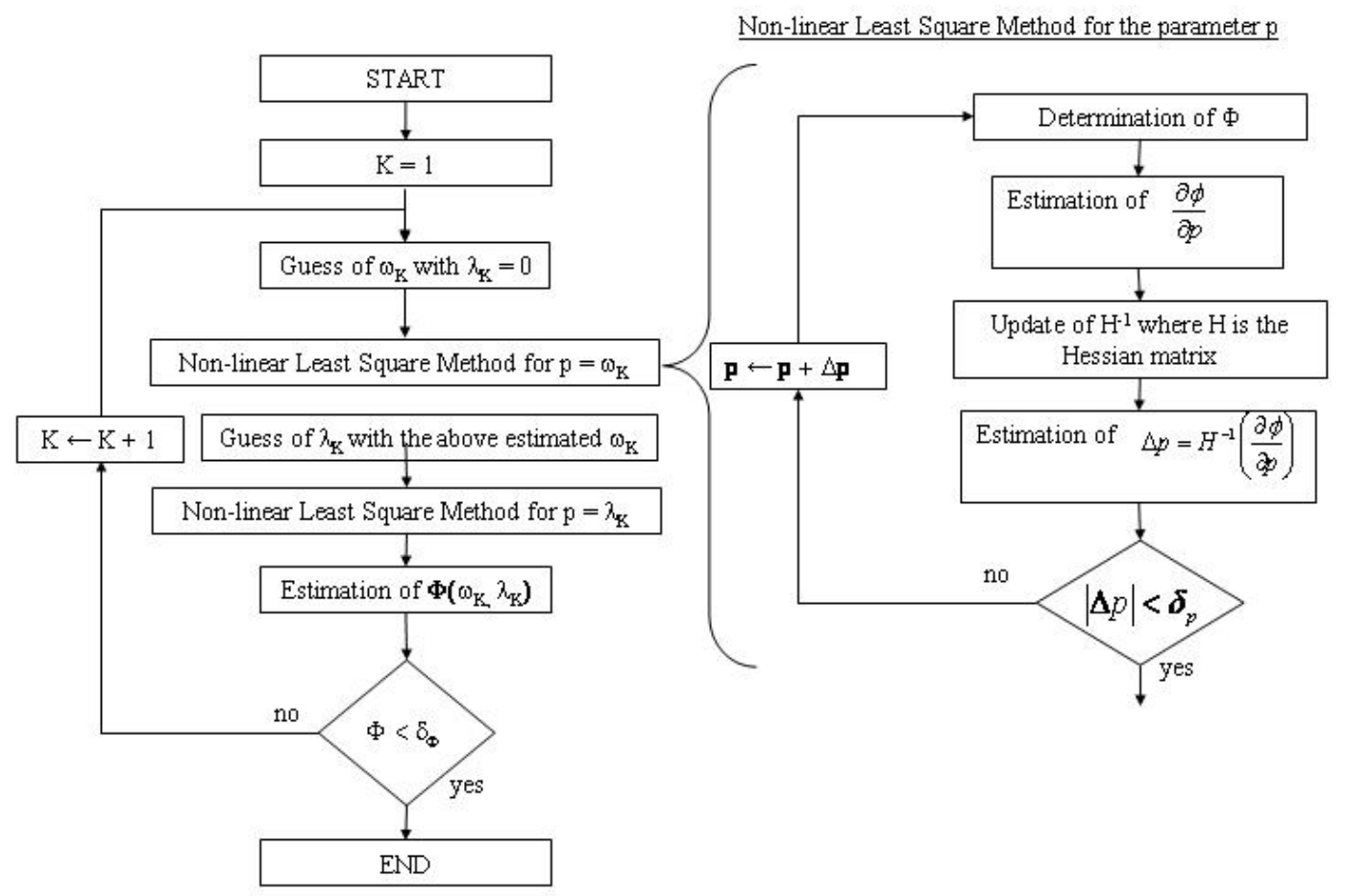

Fig. 2 

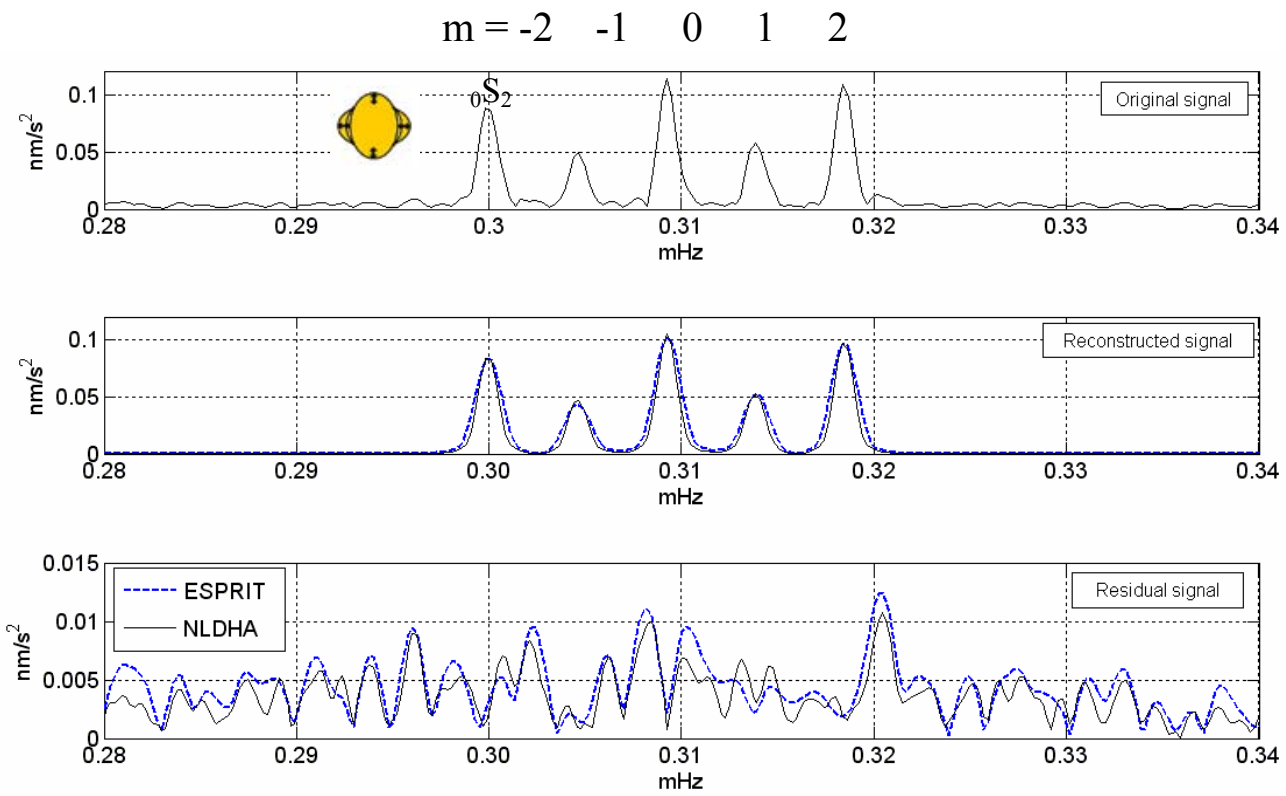

Fig. 3 (a)

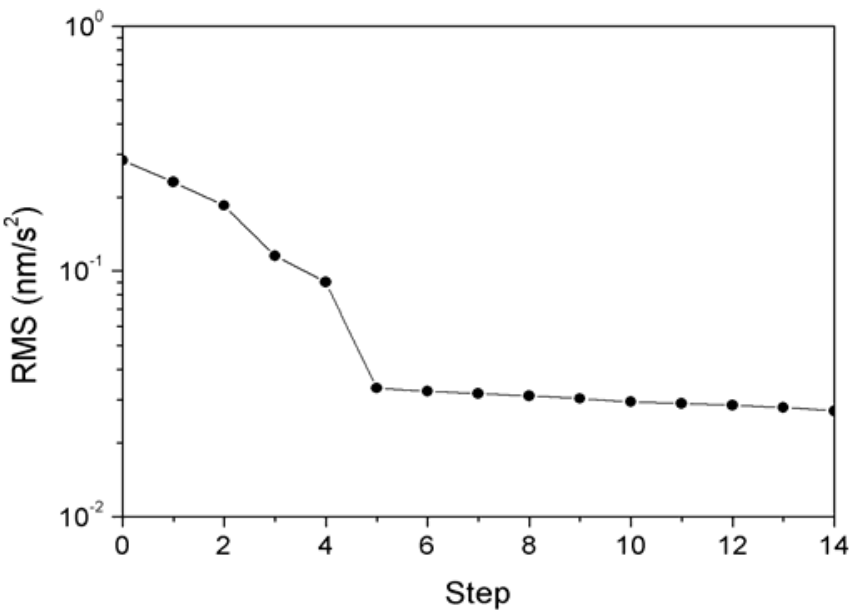

Fig. 3 (b) 

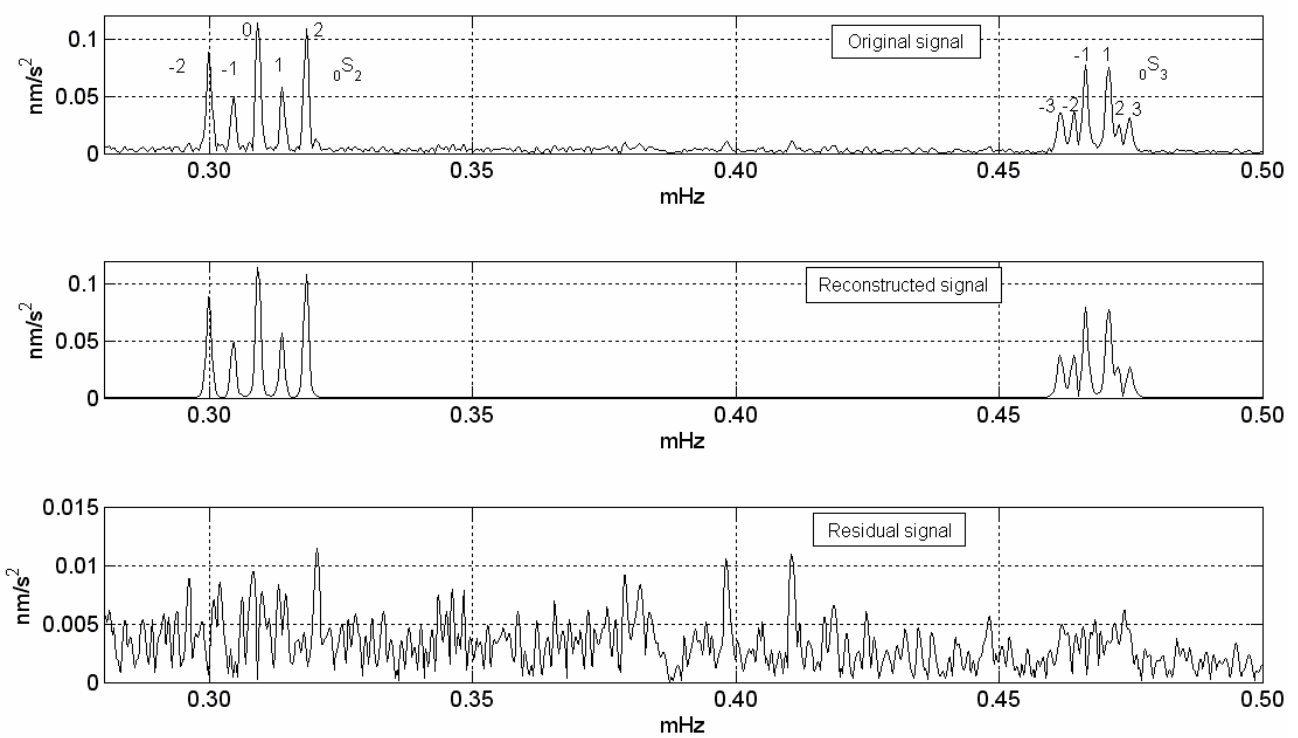

Fig. 4 (a)

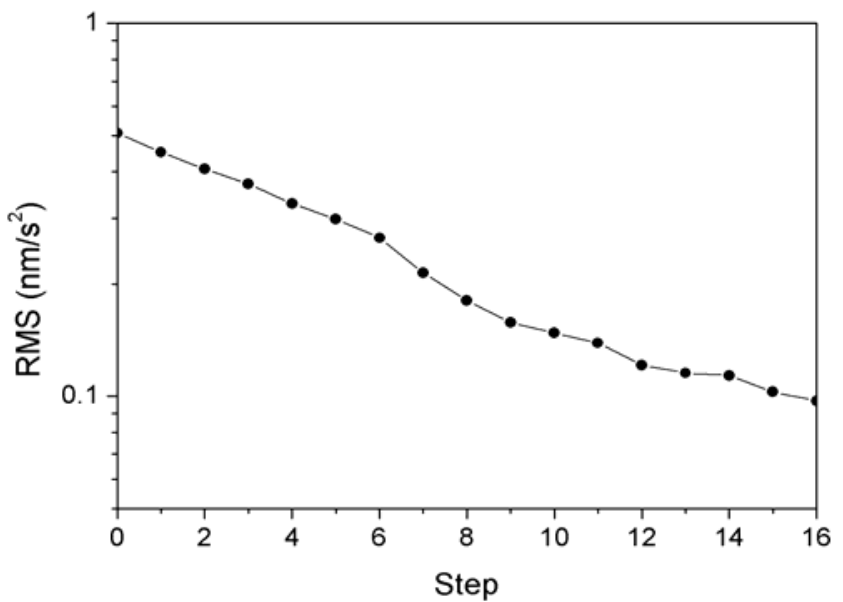

Fig. 4 (b) 

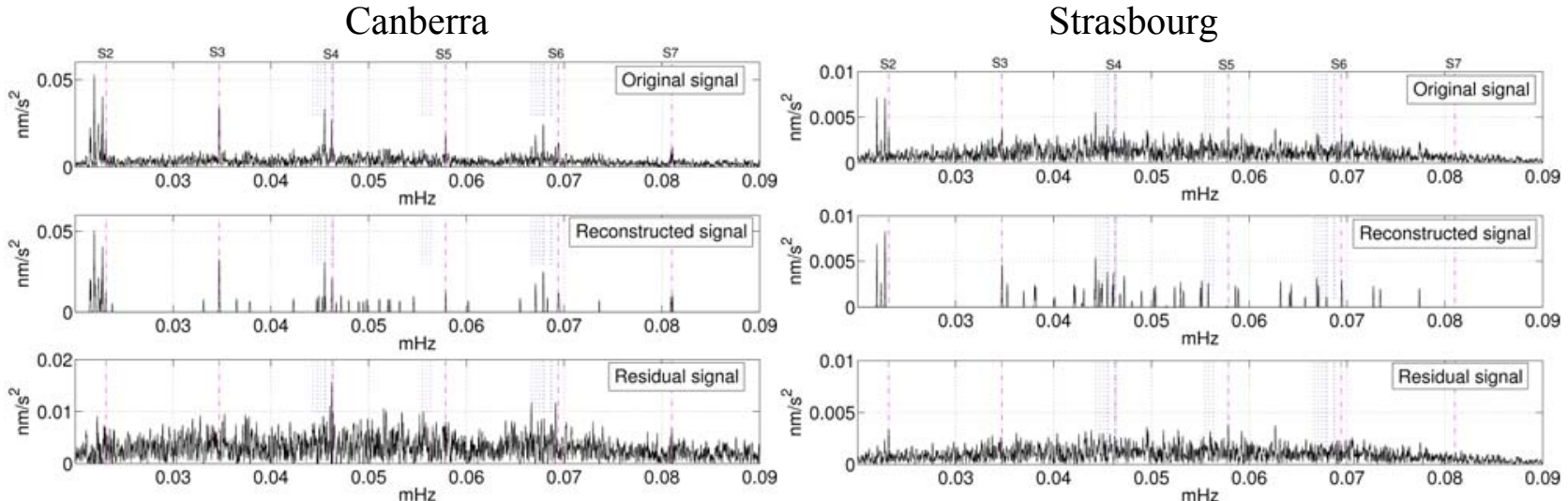

Fig. 5 (a)
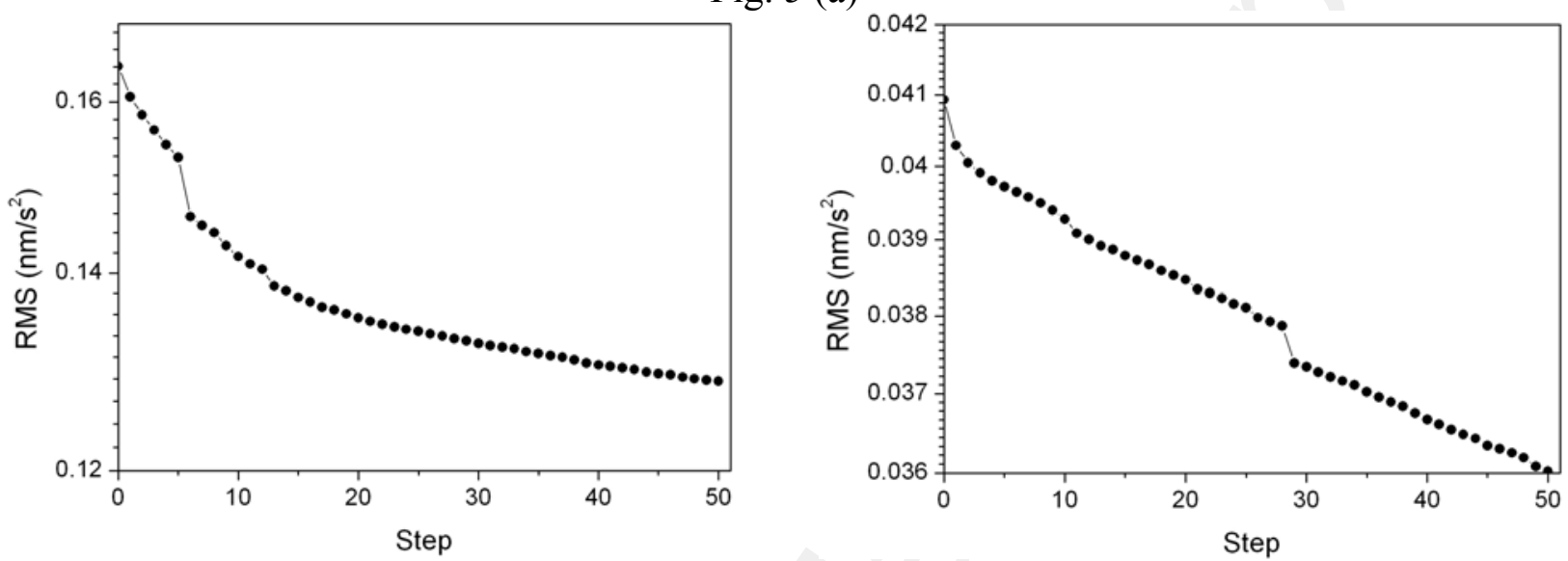

Fig. 5 (b)
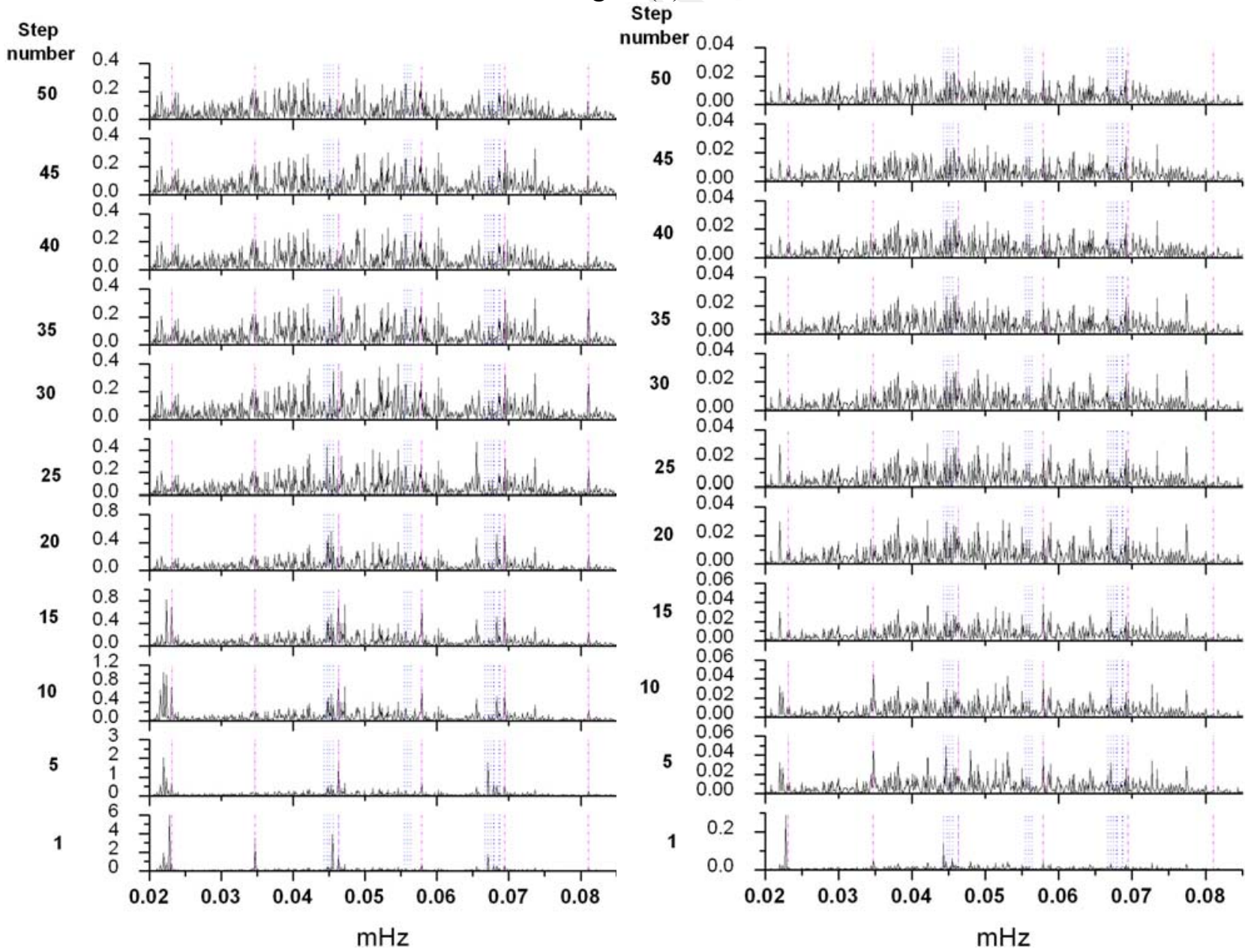

Fig. 5 (c) 